Elsevier required licence: (c) <2018>. This manuscript version is made available under the CC-BY-NC-ND 4.0 license http://creativecommons.org/licenses/by-nc-nd/4.0/

The definitive publisher version is available online at https://doi.org/10.1016/j.compeleceng.2018.05.012 


\title{
Dynamic performance improvement of an ultra-lift Luo DC-DC converter by using a Type-2 Fuzzy Neural controller
}

\author{
Amir Sharifian ${ }^{*}$, Samaneh Fathi Sasansara ${ }^{\ddagger}$, M. Jabbari Ghadi ${ }^{\#}$, Sahand Ghavidel ${ }^{\#}$, Li Li $^{\#}$, \\ Jiangfeng Zhang ${ }^{\#}$ \\ Guilan regional electric company, P.O.Box 41377-18775, Rasht, Iran. \\ $¥$ Department of Electrical Engineering, University of Guilan, P.O.Box 41996-13776, Rasht, Iran. \\ \# Faculty of Engineering and Information Technology, University of Technology Sydney, PO Box 123, \\ Broadway, NSW 2007, Australia
}

Amir.sharifiyan, Samane867@gmail.com, Mojtaba.jabbarighadi, Sahand.ghavideljirsaraie@student.uts.edu.au, Li.li, Jiangfeng.Zhang@uts.edu.au

\begin{abstract}
Due to the uncertainty associated with the structure and electrical elements of DC-DC converters and the nonlinear performance of these modules, designing an effective controller is highly complicated and also technically challenging. This paper employs a new control approach based on Type-2 fuzzy neural controller (T2FNC) in order to improve the dynamic response of an ultra-lift DC-DC converter under different operational conditions. The proposed controller can rapidly stabilize the output voltage of converter to expected values by tuning the converter switching duty cycle. This controller can tackle the uncertainties associated with the structure of converters, measured control signals and measuring devices. Moreover, a new intelligent method based on firefly algorithm is applied to tune the parameters of T2FNC. In order to demonstrate the effectiveness of the proposed control approach, the proposed controller is compared to PI and fuzzy controllers under different operational conditions. The simulation and comparison results validate the flexibility and efficiency of the proposed T2FNC.
\end{abstract}

Keywords: Type-2 fuzzy neural controller; Firefly algorithm; ultra-lift DC-DC converter; Type-2 fuzzy system; uncertainty.

* Corresponding Author: Amir Sharifian

Postal address: Guilan regional electric company, P.O.Box 41377-18775, Rasht, Iran.

Phone: +98-13-33325001, Fax: +98-13-33321067, E-mail: amir.sharifiyan@gmail.com 


\section{Introduction}

During recent years, applications of renewable energy resources have attracted significant attentions of researchers [1-6]. It is noted that DC-DC converters play a particularly important role in renewable energy generation systems [7]. This converter can provide different levels of DC voltages for various applications [8-10]. Having a constant rated output voltage under various operational conditions is the most vital requirement for DC-DC converters. In other words, the output voltage of DC-DC converter should be fixed at a constant value in steady state and transient state when the supply voltage and/or load current are changed. Therefore, optimized controllers are needed to achieve this target.

In this regard, the utilization of PI or PID controllers is considered as the most common approach to derive a simple solution. These controllers have the advantage such as simple structure, robust performance, high speed of operation and ease of implementations; however, their performances are not desirable in case of non-linear systems (such as ultra-lift DC-DC converter). Generally, the implementation and design of an efficient controller is a challenging task for nonlinear systems. This problem becomes more complicated when uncertainties of structure and electrical components of converters are considered. Therefore, it is necessary to develop a new control approach which is able to tackle the nonlinearity of systems and also to handle uncertainties associated with the converter [9]. A literature review on existing control methods for converters is shown in Table 1.

\begin{tabular}{|c|c|c|}
\hline \multicolumn{3}{|c|}{ Table 1. A literature review of used control method for converters. } \\
\hline References & Type of converter & Control method \\
\hline Ref. [8] & Dc-DC boost converter & Sliding mode control \\
\hline Ref. [9] & Boost converter & Robust regression \\
\hline Ref. [10] & DC-DC boost converter & $\begin{array}{c}\text { FPGA-based optimal robust minimal- } \\
\text { order controller }\end{array}$ \\
\hline Ref. [11] & Half-bridge isolated DC-DC converter & Fuzzy controller \\
\hline Ref. [12] & DC-DC power converters & Fuzzy logic controllers \\
\hline Ref. [13] & Boost converter & Fuzzy controller \\
\hline Ref. [14] & Boost converter & $\begin{array}{c}\text { Sliding mode fuzzy controller and } \\
\text { PID controller }\end{array}$ \\
\hline Ref. [15] & DC-DC converters & Adaptive fuzzy logic controller \\
\hline
\end{tabular}

According to Table 1, several control approaches, such as robust control, fuzzy control, intelligent control and combined methods, are used to improve the performance of converters; however, none of them is able to handle uncertainties.

Nowadays, the intelligent control methods, especially type- 2 fuzzy neural controller (T2FNC) and type-2 fuzzy controller (T2FC), are used in many areas of industrial applications [16-21]. The capability to handle the uncertainties from elements and structure of converter, measured control signals and measuring devices is the main advantage of these intelligent controllers comparedmto other controlling methods [16-21]. Considering the fact that all real world systems have certain degrees of uncertainty in their structure, the utilization of some methods like T2FNC is necessary to reduce 
uncertain effects generated by instrumentation elements, environmental noise and other external disturbances.

In this paper, we employ a new control approach based on T2FNC in order to improve the transient stability and dynamic response of an ultra-lift DC-DC converter under different operational conditions when natural system noise and uncertainties associated with electrical elements and structure of converter, measured control signals and measuring devices are considered. In additions, a novel optimization approach based on firefly algorithm is used to train the proposed T2FNC. In order to assess the performance of the T2FNC, it is compared with a fuzzy controller and a conventional PI controller in five different operating conditions. The results of simulations demonstrate higher efficiency of the T2FNC than previous methods in terms of faster transient response, less peak overshoot and better rejection of disturbances.

A summary of proposed method innovations are presented as follows:

1- We present a new control approach based on T2FNC to improve dynamic performance of an ultralift DC-DC converter.

2- The proposed controller can properly handle the uncertainties from converter elements and structure, measured control signals and measuring devices

3- A novel approach based on firefly algorithm is used to train the proposed T2FNC.

This paper is organized as follows. The state space modeling of ultra-lift DC-DC converter is presented in Section 2. In Section 3, the structure of T2FNC and the firefly algorithm are described. In Section 4, the control approach based on T2FNC for dynamic performance improvement of ultra-lift DC-DC converter is presented. Section 5 includes simulation results and detailed discussion. Finally, conclusions are outlined in Section 6.

\section{State space modeling of ultra-lift DC-DC converter}

As one of the most important types of non-isolated DC-DC converters, ultra-lift converter has different applications in various industrial fields, particularly in renewable energy systems [7]. Existing research shows that efficiency and performance of this converter is better than non-isolated DC-DC converters [22].

Designing and implementing an efficient closed-loop controller for this kind of ultra-lift converter is a difficult task; because a small change in duty ratio of converter switching will lead to significant changes in output voltage [23]. The formulation of converter mechanism is the first step for designing an efficient controller. The state space model is a common method to mathematically describe a physical system. It can describe dynamic performance of a system (such as switching converter) using computer simulations, which is very efficient in the implement and design of efficient controller. In this section the state space average model of an ultra-lift DC-DC converter is proposed. It should be noted that the state space formulation of ultra-lift DC-DC converter is taken from [7]. 


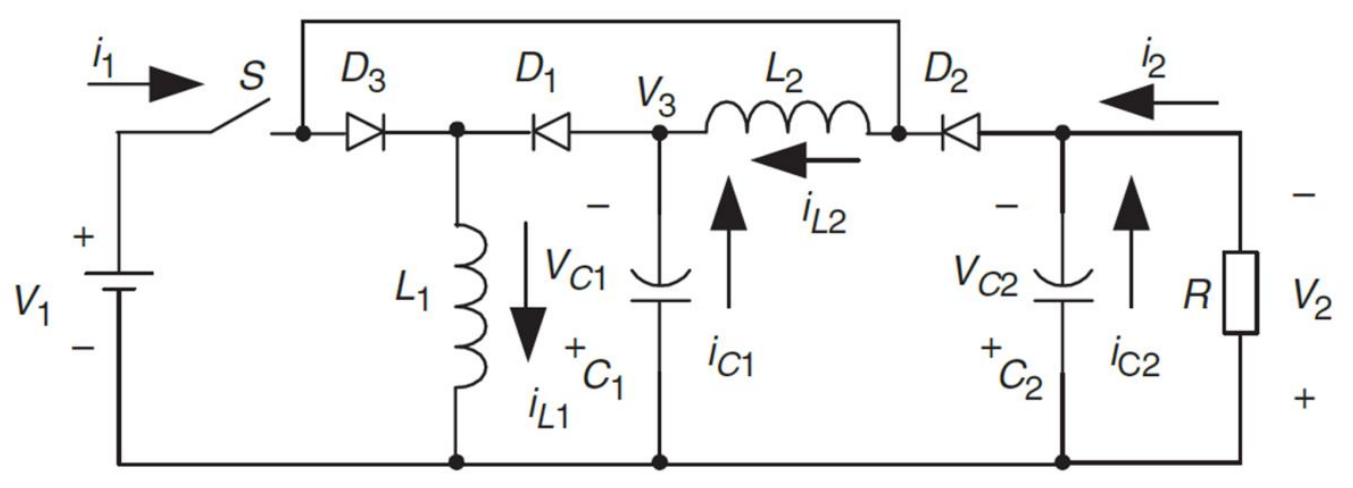

Fig. 1. Ultra-lift DC-DC converter.

As it can be seen in Fig. 1, the ultra-lift DC-DC converter performance can be described with two linear state space modes. In the first mode of the converter, switch S is ON while in the second mode the switch $\mathrm{S}$ is OFF.

Mode 1: In this mode the state equations can be described as follow:

$L_{1} \frac{d i_{L_{1}}}{d t}-V_{i n}=0$

$L_{2} \frac{d i_{L_{2}}}{d t}+v_{C_{1}}-V_{i n}=0$

$C_{1} \frac{d v_{C_{1}}}{d t}+i_{L_{2}}=0$

$C_{2} \frac{d v_{C_{2}}}{d t}+\frac{1}{R_{o}} v_{C_{2}}=0$

The state space model can be written as Eq. 2:

$\left[\begin{array}{l}\dot{i}_{L 1} \\ \dot{i}_{L 2} \\ \dot{v}_{C 1} \\ \dot{v}_{C 2}\end{array}\right]=\left[\begin{array}{cccc}0 & 0 & 0 & 0 \\ 0 & 0 & -1 / L_{2} & 0 \\ 0 & -1 / C_{1} & 0 & 0 \\ 0 & 0 & 0 & -1 / C_{2} R_{o}\end{array}\right]\left[\begin{array}{l}i_{L 1} \\ i_{L 2} \\ v_{C 1} \\ v_{C 2}\end{array}\right]+\left[\begin{array}{l}1 / L_{1} \\ 1 / L_{2} \\ 0 \\ 0\end{array}\right] V_{i n}$

Mode 2: In this mode the state equations can be described as follow: 
$L_{1} \frac{d i_{L_{1}}}{d t}-v_{C_{1}}=0$

$L_{2} \frac{d i_{L_{2}}}{d t}+v_{C_{1}}-v_{C_{2}}=0$

$C_{1} \frac{d v_{C_{1}}}{d t}+i_{L_{1}}-i_{L_{2}}=0$

$C_{2} \frac{d v_{C_{2}}}{d t}-i_{L_{2}}+\frac{1}{R_{o}} v_{C_{2}}=0$

The state space model in matrix form is given in Eq. 4:

$\left[\begin{array}{l}\dot{i}_{L 1} \\ \dot{i}_{L 2} \\ \dot{v}_{C 1} \\ \dot{v}_{C 2}\end{array}\right]=\left[\begin{array}{cccc}0 & 0 & 1 / L_{1} & 0 \\ 0 & 0 & -1 / L_{2} & 1 / L_{2} \\ -1 / C_{1} & 1 / C_{1} & 0 & 0 \\ 0 & 1 / C_{2} & 0 & -1 / C_{2} R_{o}\end{array}\right]\left[\begin{array}{l}i_{L 1} \\ i_{L 2} \\ v_{C 1} \\ v_{C 2}\end{array}\right]+\left[\begin{array}{l}0 \\ 0 \\ 0 \\ 0\end{array}\right] V_{i n}$

In order to compute the state space average model of the ultra-lift DC-DC converter, Eq. 2 and Eq. 4 are combined. Hence, the state space average model of an ultra-lift DC-DC converter is obtained following reference [7]:

$\dot{X}=A_{1} X+B_{1} V_{\text {in }} \quad$ when switch is ON (during $\left.\mathrm{d} \times \mathrm{T}_{\mathrm{s}}\right)$

$\dot{X}=A_{2} X+B_{2} V_{\text {in }} \quad$ when switch is $\operatorname{OFF}\left(\right.$ during $\left.(1-\mathrm{d}) \times \mathrm{T}_{\mathrm{s}}\right)$

$\dot{X}=\left[A_{1} \times d+A_{2} \times(1-d)\right] X+\left[B_{1} \times d+B_{2} \times(1-d)\right] V_{i n}$

$\left[\begin{array}{l}\dot{i}_{L 1} \\ \dot{i}_{L 2} \\ \dot{v}_{C 1} \\ \dot{v}_{C 2}\end{array}\right]=\left[\begin{array}{cccc}0 & 0 & (1-d) / L_{1} & 0 \\ 0 & 0 & -1 / L_{2} & (1-d) / L_{2} \\ (d-1) / C_{1} & (1-2 d) / C_{1} & 0 & 0 \\ 0 & (1-d) / C_{2} & 0 & -1 / C_{2} R_{o}\end{array}\right]\left[\begin{array}{l}i_{L 1} \\ i_{L 2} \\ v_{C 1} \\ v_{C 2}\end{array}\right]+\left[\begin{array}{l}d / L_{1} \\ d / L_{2} \\ 0 \\ 0\end{array}\right] V_{\text {in }}$

$y=\left[\begin{array}{l}V_{o} \\ i_{i}\end{array}\right]=\left[\begin{array}{llll}0 & 0 & 0 & 1 \\ d & d & 0 & 0\end{array}\right]\left[\begin{array}{l}i_{L 1} \\ i_{L 2} \\ v_{C 1} \\ v_{C 2}\end{array}\right]$

where $X$ is a matrix including the state variables, $T_{s}$ is the switching period of the converter, and $d$ is the duty cycles of the converter switch.

It should be mentioned that Eq. 1 and Eq. 3 are linear ordinary differential equations. Equation. 1 describes the converter behavior when the switch is ON and Eq. 3 describes the system when the switch is OFF. Since the state space average equation of the converter depends on the state of the switching element (duty cycle), state space equation of the ultra-lift DC-DC converter is nonlinear (Eq. $8)$. 
Therefore, due to the nonlinear behavior of the ultra-lift DC-DC converter, we need a nonlinear controller for better performance. In this paper a T2FN controller is used.

\section{1. Type-2 Fuzzy Neural Network}

The detail of a Type-2 fuzzy Gaussian membership function is illustrated in Fig. 2.

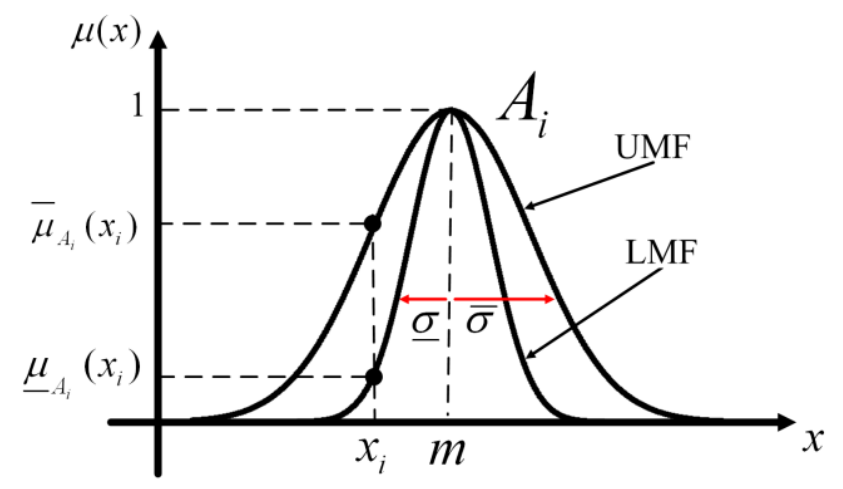

Fig. 2. The structure of a Type-2 Gaussian membership function.

In Fig. 2, $\underline{\mu}_{A_{i}}\left(x_{i}\right), \bar{\mu}_{A_{i}}\left(x_{i}\right),[\bar{\sigma}, \underline{\sigma}]$ and $m$ are the lower membership function (LMF), upper membership function (UMF), standard deviation and the mean values of Type-2 fuzzy Gaussian membership function, respectively. The distance between superior membership function and inferior membership function determinates the footprint of uncertainty (FOU). The value of membership degree can be computed as follows:

$\bar{\mu}_{A}(x)=\exp \left(-\frac{1}{2} \frac{(x-m)^{2}}{\bar{\sigma}^{2}}\right)$

$\underline{\mu}_{A}(x)=\exp \left(-\frac{1}{2} \frac{(x-m)^{2}}{\underline{\sigma}^{2}}\right)$

The data processing method and structure of the type-2 fuzzy neural network is shown in Fig. 3. 


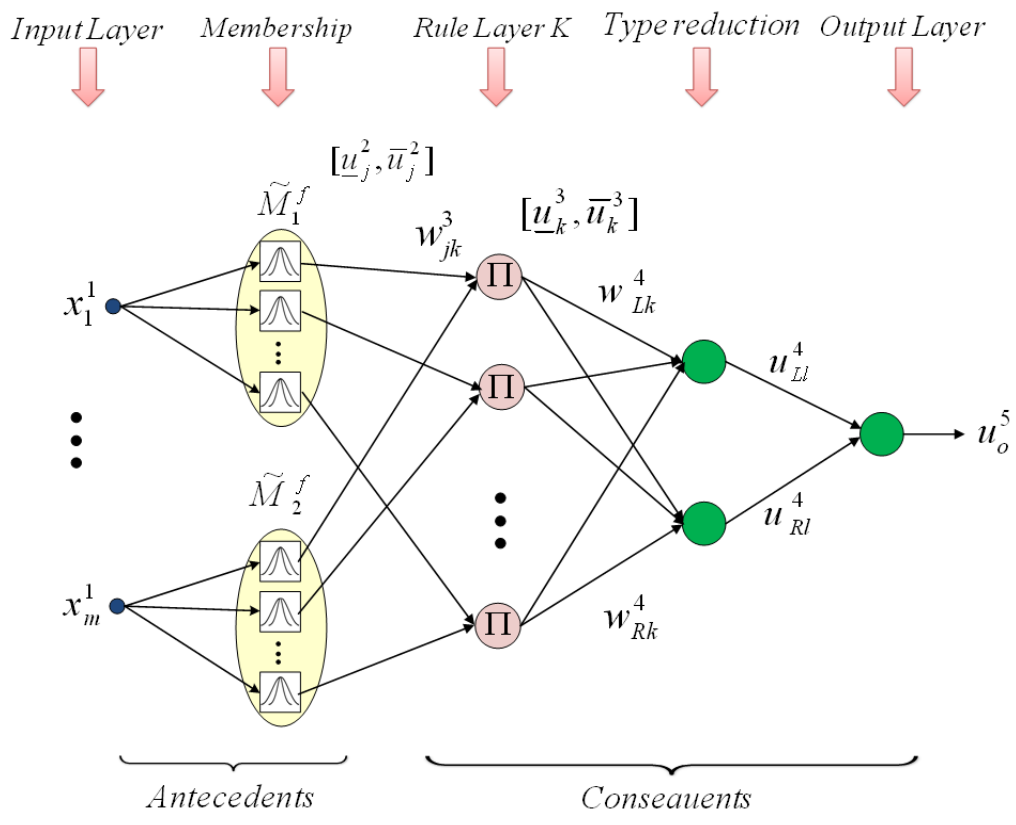

Fig. 3. Structure of the type-2 fuzzy neural network.

According to Fig.3, the proposed Type-2 fuzzy neural network is a combination of a type-2 fuzzy linguistic process and a neural network. The IF-THEN rule for this network can be obtained as follow:

$R_{f}:$ IF $x_{1}^{1}$ is $\tilde{M}_{1}^{f}$, and $x_{2}^{1}$ is $\tilde{M}_{2}^{f}$, and $\ldots$ and $x_{m}^{1}$ is $\tilde{M}_{m}^{f}$

THEN $u_{1}$ is $\left[w_{R f}^{4}, w_{L f}^{4}\right]$

where $\tilde{M}_{m}^{f}, f, x_{m}^{1}$ and $\left[w_{R f}^{4}, w_{L f}^{4}\right]$ are type-2 fuzzy sets, number of rules, the inputs of the network and set of weights, respectively. The data processing in type-2 fuzzy neural network can be explained by the following steps:

Step 1 (Input layer): For input variable $i$ in this layer, the output can be computed as follows:

$\operatorname{net}_{i}^{1}(N)=x_{i}^{1}, u_{i}^{1}=f_{i}^{1}\left(\operatorname{net}_{i}^{1}(N)\right)=\operatorname{net}_{i}^{1}(N), i=1,2$

where $N$ is the number of iterations.

Step 2 (Membership layer): There is a type-2 fuzzy set in this layer dose. For the jth input in this step, the output can be computed as Eq. 13.

$u_{j}^{2}=\tilde{M}_{i}^{j}\left(x_{i}^{2}\right)=f_{j}^{2}\left(n e t_{j}^{2}(N)\right)=\exp \left(n e t_{j}^{2}(N)\right)=\left\{\begin{array}{lll}\bar{u}_{j}^{2}(N) & \text { as } & \sigma_{i j}=\bar{\sigma}_{i j} \\ \underline{u}_{j}^{2}(N) & \text { as } & \sigma_{i j}=\underline{\sigma}_{i j}\end{array} \quad j=1, \ldots, s\right.$

$\operatorname{net}_{j}^{2}(N)=-\frac{1}{2}\left(\frac{x_{i}^{2}-m_{i j}}{\sigma_{i j}}\right)^{2}$

where $s, m_{i j}$ and $\sigma_{i j}$ are the number of the linguistic values, the mean and the standard deviation values of the Gaussian membership function, respectively.

Step 3 (Rule layer): In this layer, for the $k$ th rule node can be written: 
$u_{k}^{3}(N)=f_{k}^{3}\left(n e t_{k}^{3}(N)\right)=n e t_{k}^{3}(N)\left\{\begin{array}{l}\bar{u}_{k}^{3}(N)=\prod_{j=2}^{n} w_{j k}^{3} \bar{u}_{j}^{2} \\ \underline{u}_{k}^{3}(N)=\prod_{j=1}^{n} w_{j k}^{3} \underline{u}_{j}^{2}\end{array} \quad k=1, \ldots, n\right.$

where $x_{j}^{3}, w_{j k}^{3}$ and $n$ are $j$ th input to step 3, weights between the membership layer and rule layer and number of rules, respectively.

Step 4 (Type-reduction layer): In this step, the Karnik-Mendel (KM) algorithm [24, 25] is applied for dimension reduction. The output value in this step can be written as:

$n e t_{l}^{4}(N)=\frac{\sum_{k=1}^{n} w_{k}^{4} u_{k}^{3}(N)}{\sum_{k=1}^{n} u_{k}^{3}(N)}$

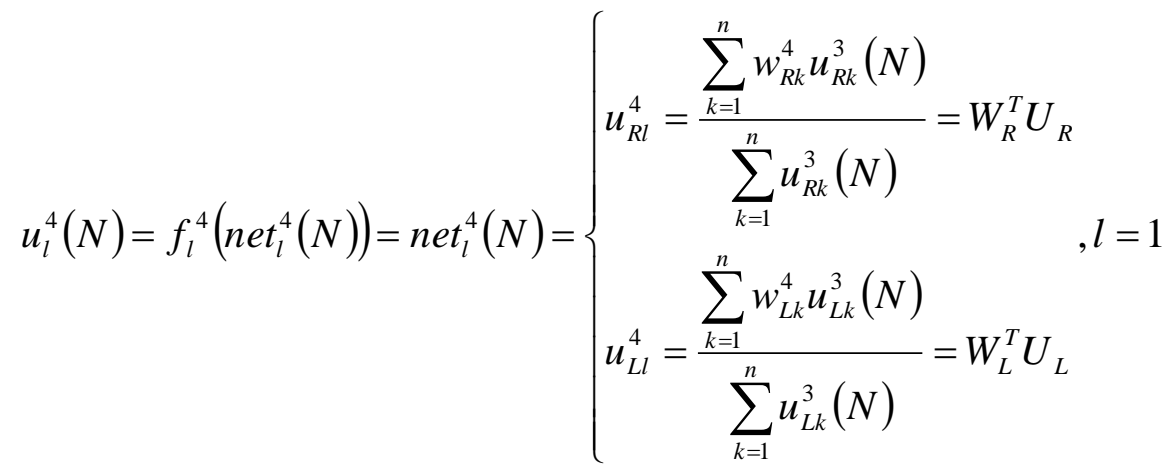

where $w_{k}^{4} \in\left[w_{R k}^{4} w_{L k}^{4}\right]$ is the centroid of the obtained type- 2 set.

$$
\begin{aligned}
& W_{R}=\left[w_{R 1}^{4} w_{R 2}^{4} \ldots w_{R n}^{4}\right]^{T} \\
& W_{L}=\left[w_{L 1}^{4} w_{L 2}^{4} \ldots w_{L n}^{4}\right]^{T} \\
& U_{R}=\left[\frac{u_{R 1}^{3}(N)}{\sum_{k=1}^{n} u_{R k}^{3}(N)} \frac{u_{R 2}^{3}(N)}{\sum_{k=1}^{n} u_{R k}^{3}(N)} \ldots \frac{u_{R n}^{3}(N)}{\sum_{k=1}^{n} u_{R k}^{3}(N)}\right]^{T} \\
& U_{L}=\left[\frac{u_{L 1}^{3}(N)}{\sum_{k=1}^{n} u_{L k}^{3}(N)} \frac{u_{L 2}^{3}(N)}{\sum_{k=1}^{n} u_{L k}^{3}(N)} \ldots \frac{u_{L n}^{3}(N)}{\sum_{k=1}^{n} u_{L k}^{3}(N)}\right]^{T}
\end{aligned}
$$

Step 5 (Output layer): In this step the values of $u_{L l}^{4}$ and $u_{R l}^{4}$ are combined in order to compute the output value. The output value in this step can be computed as: 
$u_{o}^{5}=\frac{u_{R l}^{4}+u_{L l}^{4}}{2}=\frac{1}{2}\left(W_{R}^{T} U_{R}+W_{L}^{T} U_{L}\right)=\frac{1}{2} W^{T} U\left(X^{1}, m, \sigma\right)$

$W=\left[W_{R}^{T} W_{L}^{T}\right]^{T}$

$U=\left[U_{R} U_{L}\right]^{T}$

$X^{1}=\left[x_{1}^{1} x_{2}^{1}\right]$

$m=\left[\begin{array}{ll}m_{11} \ldots m_{1 s} & m_{21} \ldots m_{2 s}\end{array}\right]$

$\sigma=\left[\begin{array}{lll}\sigma_{11} \ldots \sigma_{1 s} & \sigma_{21} \ldots \sigma_{2 s}\end{array}\right]$

where $u_{o}^{5}$ is the type-2 fuzzy neural network output.

The Type-2 fuzzy neural network requires the adjustment of its parameters for all the layers such that the output of the network can approach desired output. There are several algorithms such as gradient descent, Levenberg-Marquardt and resilient back-propagation for training the intelligent neural network systems. Hence, selecting an efficient training algorithm is essential for desirable performance of Type-2 fuzzy neural network. In this paper we use a new training method based on firefly optimization algorithm to train Type-2 fuzzy neural network more accurately. Details of the proposed method are described in next sections. The target function for training process can be defined by Eq. 18:

$J=\frac{1}{2}\left(y_{d}-y\right)^{2}$

The firefly algorithm is applied to minimize the target function (Eq. 18) in order to achieve expected performance of T2FNC.

\section{2. Firefly algorithm:}

During recent years, a large number of optimization algorithms have been presented for different aspects of power system research [26-29]. The firefly algorithm that has firstly been presented in [**], is a nature-inspired searching algorithm. In fact, this optimization algorithm is inspired by mating manner of firefly [30]. Fireflies have the capability of generating a cold light (bioluminescence phenomenon) owing to their particular photogenic capabilities. This light simplifies the task for drawing their potential prey, finding mates and defending themselves. The fireflies have tendency to waggle toward brighter locations. This fact is similar to find efficient optimal solutions in optimization problems. The brighter firefly in the population attracts other fireflies. The speed or traction a firefly towards another firefly appertains to the attractiveness of destination firefly. Besides, the attractiveness appertains to the comparative interval between fireflies which is a function of fireflies' brightness. The firefly attractiveness function can be computed as follows [30]:

$$
\beta(r)=\beta_{0} \times \exp \left(-r^{m}\right), \text { with } m \geq 1
$$


$r_{i j}=\left\|x_{i}-x_{j}\right\|=\sqrt{\sum_{k=1}^{d}\left(x_{i, k}-x_{j, k}\right)^{2}}$

where $r, \gamma$ and $\beta_{0}$ are distance between any two fireflies, absorption coefficient and the initial attractiveness at $r=0$, respectively. The brightness and location of the fireflies are updated in each algorithm iteration when the fireflies move towards the brighter area. The $i$ th firefly motion which is engrossed by a more absorbing (i.e. brighter) firefly $j$ is described as follow [30]:

$x_{i}=x_{i}+\beta_{0} e^{-\gamma r_{i j}^{2}}\left(x_{i}-x_{j}\right)+\alpha(\operatorname{rand}-0.5)$

where $x_{i}, \beta_{0} e^{-\gamma r_{i j}^{2}}\left(x_{i}-x_{j}\right), \alpha($ rand -0.5$)$ and $\alpha$ are firefly current position, the firefly attractiveness seen by adjoining fireflies, firefly's random motion and a randomization parameter $(\alpha \in[0,1])$, respectively. The attractiveness and brightness of each firefly are calculated at each iteration of the algorithm. For each firefly, the value of brightness is compared with that of other fireflies; then fireflies' location are updated using Eq. 21. After an enough number of algorithm iterations, the optimization process is terminated and the best available solution is returned [30]. The flowchart of firefly algorithm is illustrated in Fig. 4. 


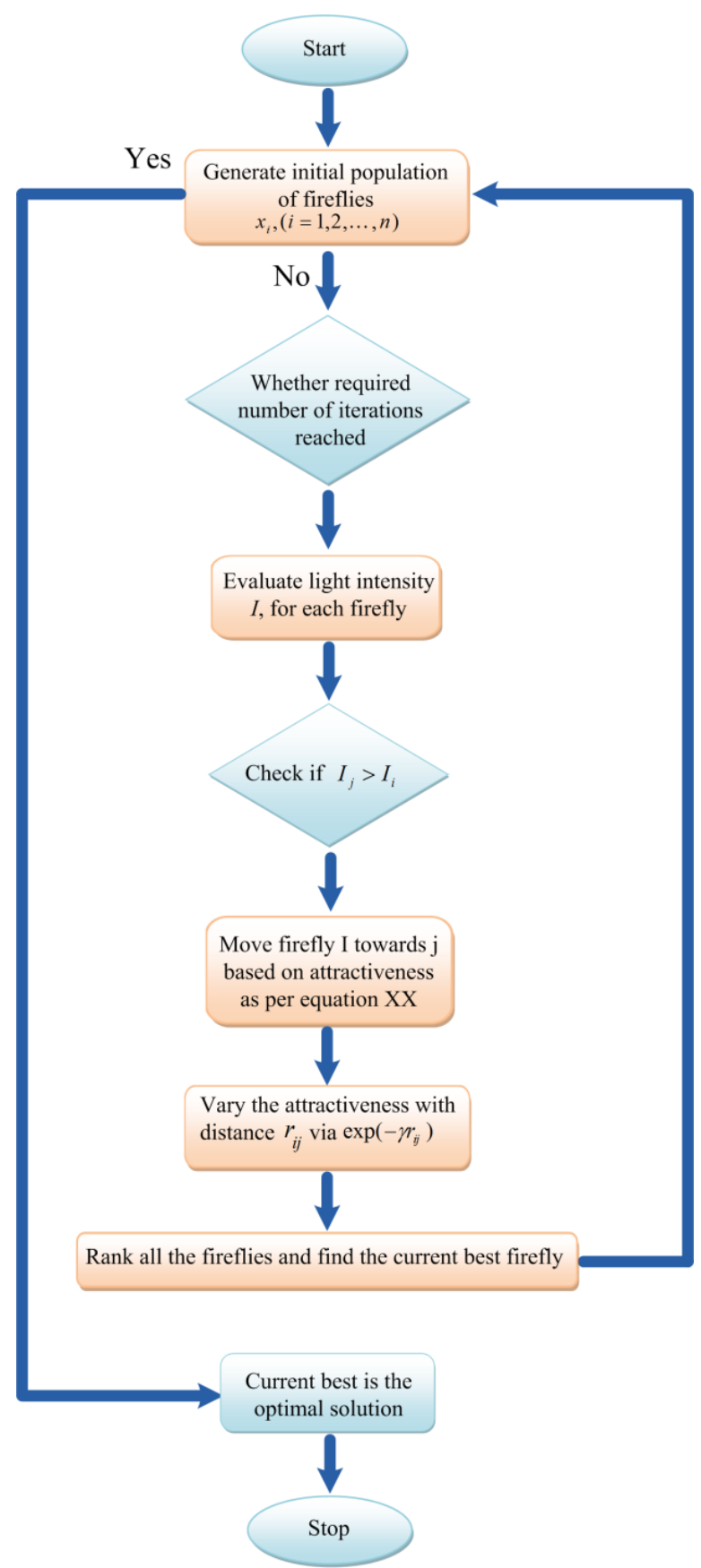

Fig. 4. Flowchart of the firefly algorithm.

\section{Proposed method based on Type-2 Fuzzy Neural controller}

The main purpose of this section is to design an efficient controller for an ultra-lift DC-DC converter. In addition, the designed controller should be able to handle the uncertainties from electrical elements and structure of converter, measured control signals and measuring devices.

The detailed structure of T2FNC is illustrated in Fig. 5. 


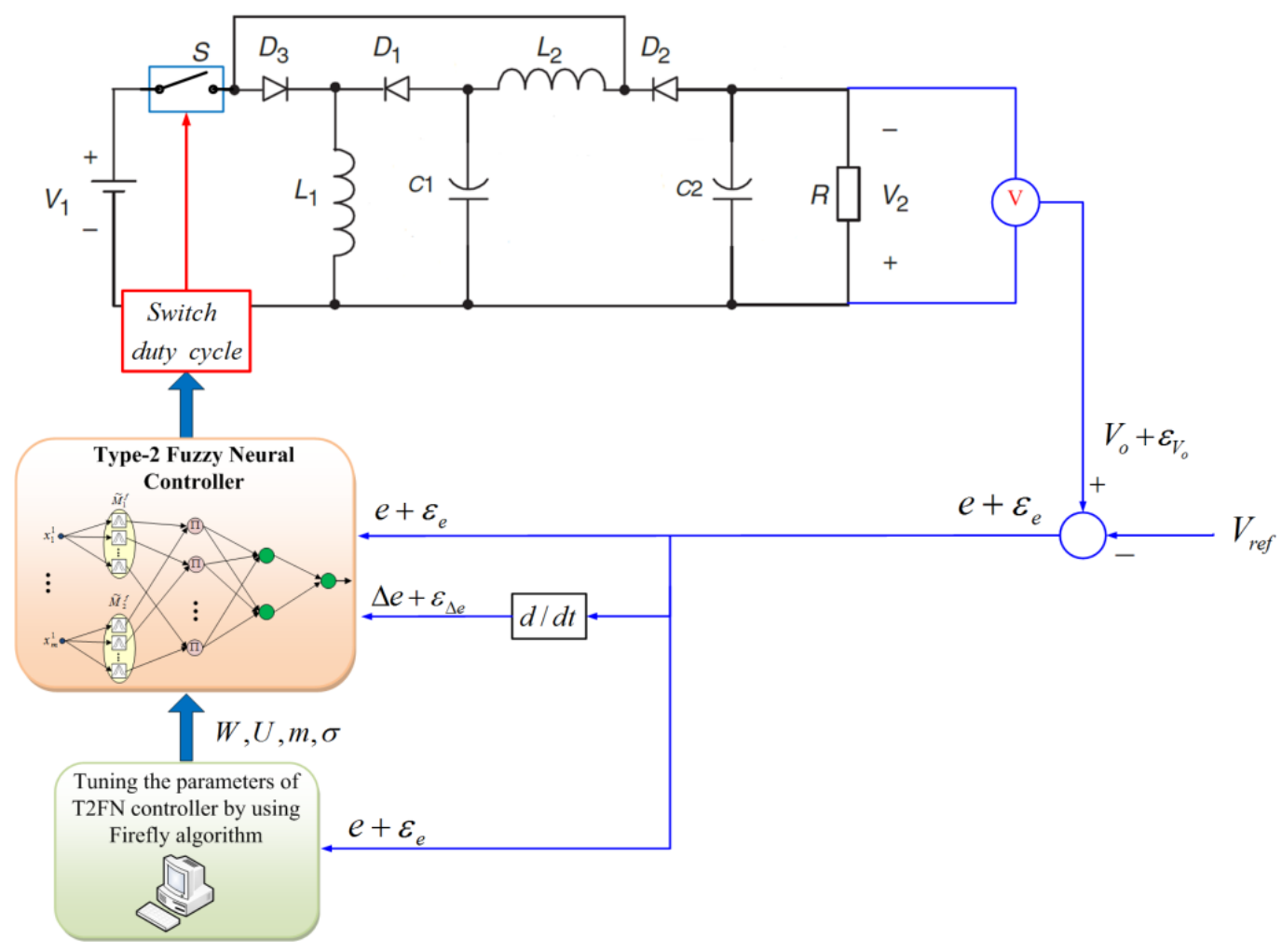

Fig. 5. Proposed Type-2 Fuzzy Neural controller (T2FNC).

As illustrated in Fig. 5, the T2FN controller fixes the output voltage of converter by tuning the converter duty cycle of switching. A voltmeter is used to compute the value of converter output voltage. The measured output voltage of converter is compared with a reference voltage in order to calculate the value of error signal $(e)$. Then, a derivative block is applied to compute the value of change in error signal $(\Delta e)$. The values of error signal $(e)$ and change in error signal $(\Delta e)$ are used for tuning the converter duty cycle of switching.

According to Fig. 5, the control scheme is composed of two main blocks. The first block is a Type-2 fuzzy neural controller. This block has two inputs (the error signal $(e)$ and the change in error signal $(\Delta e))$ and an output which is the switching duty cycle. In our proposed control method, the T2FNC uses the inputs control signals ( $e$ and $\Delta e$ ) in order to regulate the converter output voltage by tuning the switching duty cycle. The operation detail of this block has been explained in the pervious section.

The second block is a training block. In this block, a new method based on firefly optimization algorithm is used for tuning the parameters of T2FNC by using the error signal $(e)$. The input signal of training block is the values of error signal $(e)$ and its output signals are the weighting interval factors and also the mean and standard deviation of the T2FNC.

It is noted that the values of measured output voltage $\left(V_{o}\right)$ and control signals $(e$ and $\Delta e$ ) have some degrees of uncertainty and noise. These uncertainties associated with $V_{o}, e$ and $\Delta e$ are illustrated by $\varepsilon_{V_{o}}, \varepsilon_{e}$ and $\varepsilon_{\Delta e}$ in Fig. 5, respectively. The T2FN fuzzy neural network can properly handle such uncertainties. The mechanism of T2FN controller to handle uncertainties with control signals ( $e$ and $\Delta e$ ) is illustrated in Fig. 6. 


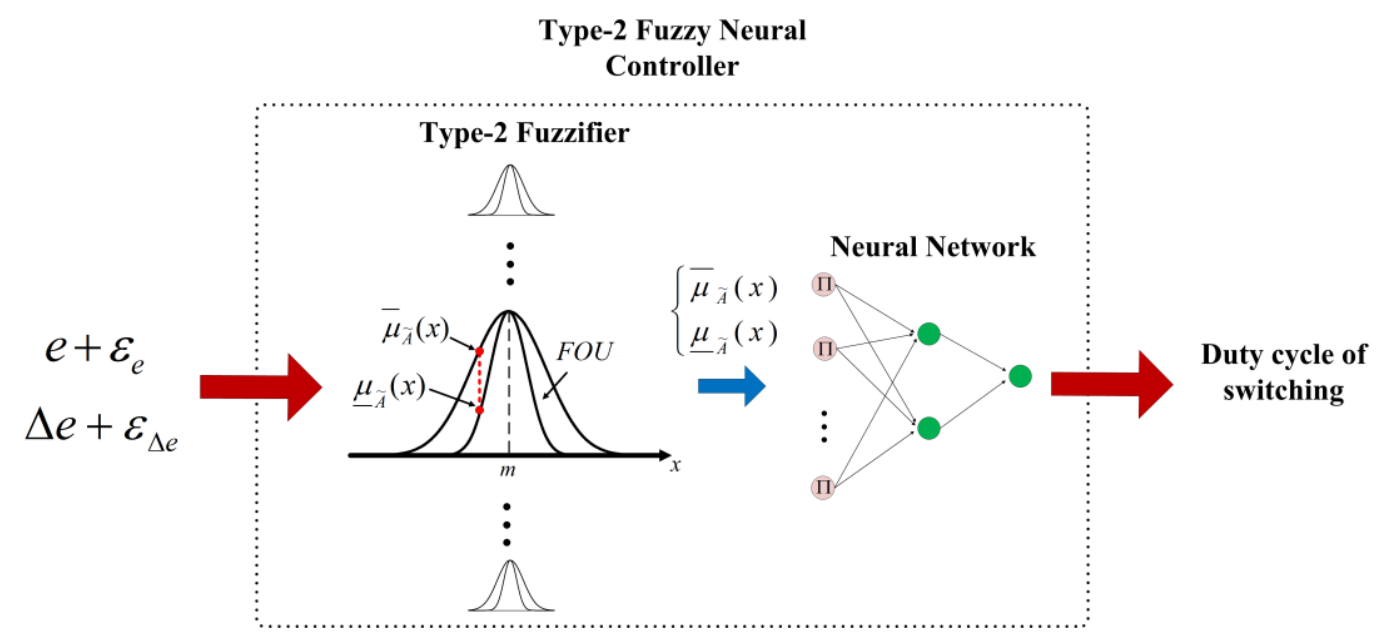

Fig. 6. The mechanism of T2FN controller in orders to handle uncertainties with control signals.

According to Fig. 6, the proposed T2FN controller is composed of two parts including a set of Type2 Fuzzifiers and a neural network. The Type-2 Fuzzifiers are used to model the uncertainties of inputs. The Type-2 Fuzzifiers transform the control signals along with their uncertainties into Type-2 fuzzy sets. The outputs of Type-2 Fuzzifiers are values of $\underline{\mu}_{A_{i}}\left(x_{i}\right)$ and $\bar{\mu}_{A_{i}}\left(x_{i}\right)$. Then, the values of $\underline{\mu}_{A_{i}}\left(x_{i}\right)$ and $\bar{\mu}_{A_{i}}\left(x_{i}\right)$ are applied as inputs of neural network. In other words, the intelligent systems use fuzzified control signals $\left(\underline{\mu}_{A_{i}}\left(x_{i}\right)\right.$ and $\left.\bar{\mu}_{A_{i}}\left(x_{i}\right)\right)$ instead of control signals $(e$ and $\Delta e)$ that contain uncertainties.

Hence, the T2FNC controller can properly handle the uncertainties associated with converter's structure, measured control signals and measuring devices and improve dynamic performance of an ultra-lift DC-DC converter.

\section{The simulation results of T2FN PI controller}

In this section, the proposed controlling method in pervious sections is simulated, then the simulation results \are compared with other conventional controlling approaches (i.e. fuzzy controller and PI).

The first step is to simulate ultra-lift DC-DC converter and proposed T2FN controller. In this paper, the MATLAB software is used to achieve this goal. The simulated system is illustrated in Fig.7. 


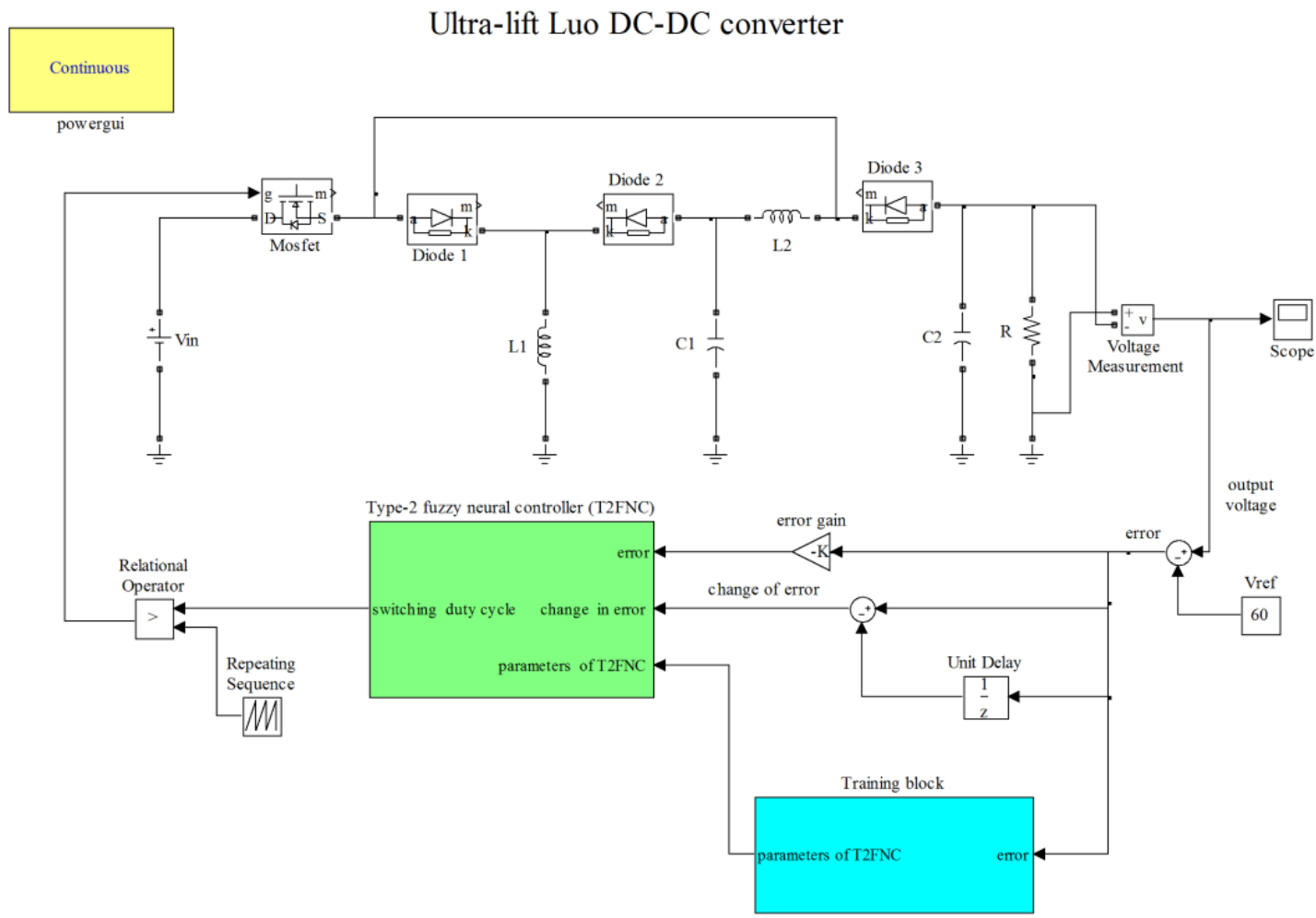

Fig. 7. The simulated system in MATLAB.

The value of converter circuit elements are given in Table 2 [22].

Table 2. The values of converter circuit element [31,32].

\begin{tabular}{|c|c|c|c|}
\hline circuit parameter & value & circuit parameter & value \\
\hline $\mathrm{V}_{1}$ (input voltage) & $10 \mathrm{~V}$ & $\mathrm{~L}_{1}, \mathrm{~L}_{2}$ & $1 \mathrm{mH}$ \\
\hline $\mathrm{V}_{\text {ref }}$ (reference voltage) & $60 \mathrm{~V}$ & $\mathrm{C}_{1}, \mathrm{C}_{2}$ & $1 \mu \mathrm{F}$ \\
\hline $\mathrm{f}_{\mathrm{S}}$ (switching frequency) & $50 \mathrm{KHz}$ & $\mathrm{R}$ & $3 \mathrm{~K} \Omega$ \\
\hline
\end{tabular}

The design process of T2FNC is explained in the following steps:

Step 1: Determine input variables. In this paper, values of the error signal $(e)$ and the change in error signal $(\Delta e)$ are used as input variable of T2FNC.

Step 2: According to Step 1, four type-2 fuzzy sets are assumed for each input variable of T2FNC. Here, Gaussian membership functions are chosen for these input variables.

Step 3: Firefly algorithm is applied for optimal parameter tuning in T2FNC. These parameters include the weighting interval factor as well as values for mean and standard deviation of the T2FNC. The parameters for firefly algorithm are shown in Table 3.

Table 3. The supposed parameters for firefly algorithm.

\begin{tabular}{|c|c|c|c|}
\hline FA parameter & value & FA parameter & value \\
\hline $\begin{array}{c}\text { Number of fireflies or } \\
\text { population }\end{array}$ & 20 & Randomization parameter $\alpha$ & 0.25 \\
\hline Iterations or generations & 400 & Attractiveness $\beta_{0}$ & 0.20 \\
\hline
\end{tabular}


The performance and effectiveness of the proposed T2FN controller to improve dynamic response of the ultra-lift DC / DC converter is investigated. The performance of T2FNC is simulated in five different operating points as Table 4. In addition, the T2FNC is compared with fuzzy controller [11] and PI controller in order to demonstrate the efficiency and accuracy of the proposed methodology. The simulation results are shown in Figs. 8-12.

Table 4. Five different operating points for performance evaluation

\begin{tabular}{|c|c|c|c|c|}
\hline Condition number & Operating condition & $\mathbf{V}_{\text {in }}(\mathbf{V})$ & Io $_{(\mathbf{m A})}$ & $\mathbf{R}(\mathbf{K} \Omega)$ \\
\hline 1 & Nominal condition & 10 & 3.33 & 3 \\
\hline 2 & Max $\mathrm{V}_{\text {in }}$ and Max $\mathrm{I}_{\mathrm{O}}$ & 13 & 8.66 & 1.5 \\
\hline 3 & Max $\mathrm{V}_{\text {in }}$ and Min $\mathrm{I}_{\mathrm{O}}$ & 13 & 2.16 & 6 \\
\hline 4 & Min $\mathrm{V}_{\text {in }}$ and Max $\mathrm{I}_{\mathrm{O}}$ & 6 & 4 & 1.5 \\
\hline 5 & Min $\mathrm{V}_{\text {in }}$ and Min $\mathrm{I}_{\mathrm{O}}$ & 6 & 1 & 6 \\
\hline
\end{tabular}

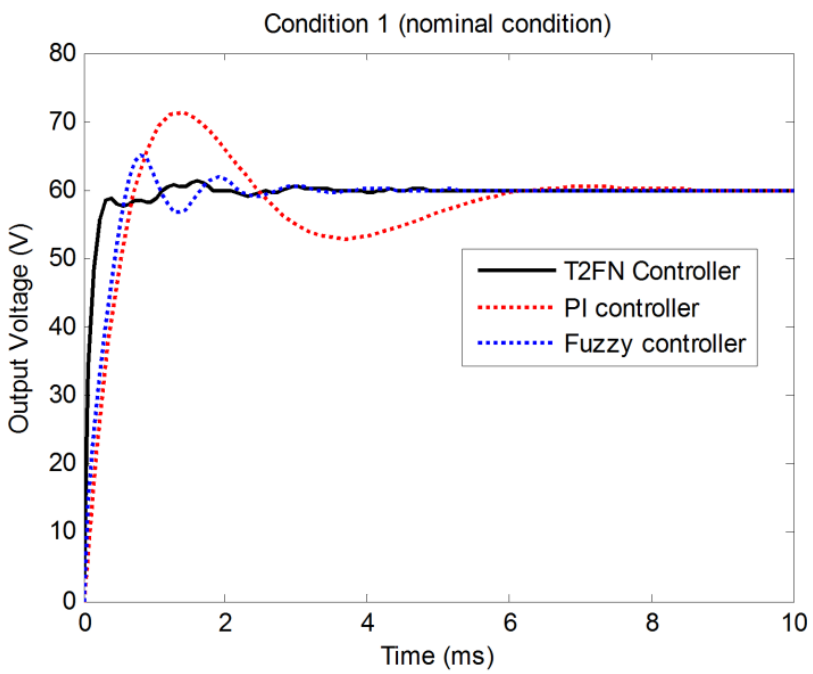

Fig. 8. Response for condition $1\left(V_{\mathrm{in}}=10 \mathrm{v}, \mathrm{I}_{\mathrm{o}}=3.33 \mathrm{~mA}, \mathrm{R}=3 \mathrm{~K} \Omega\right)$

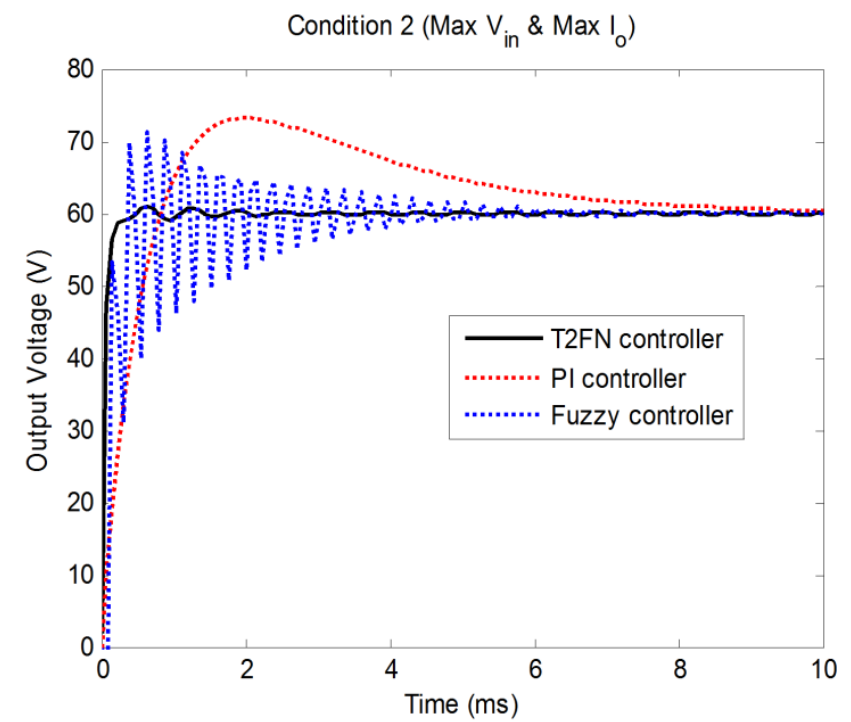

Fig. 9. Response for condition $2\left(\mathrm{~V}_{\mathrm{in}}=13 \mathrm{v}, \mathrm{I}_{\mathrm{o}}=8.66 \mathrm{~mA}, \mathrm{R}=1.5 \mathrm{~K} \Omega\right)$ 


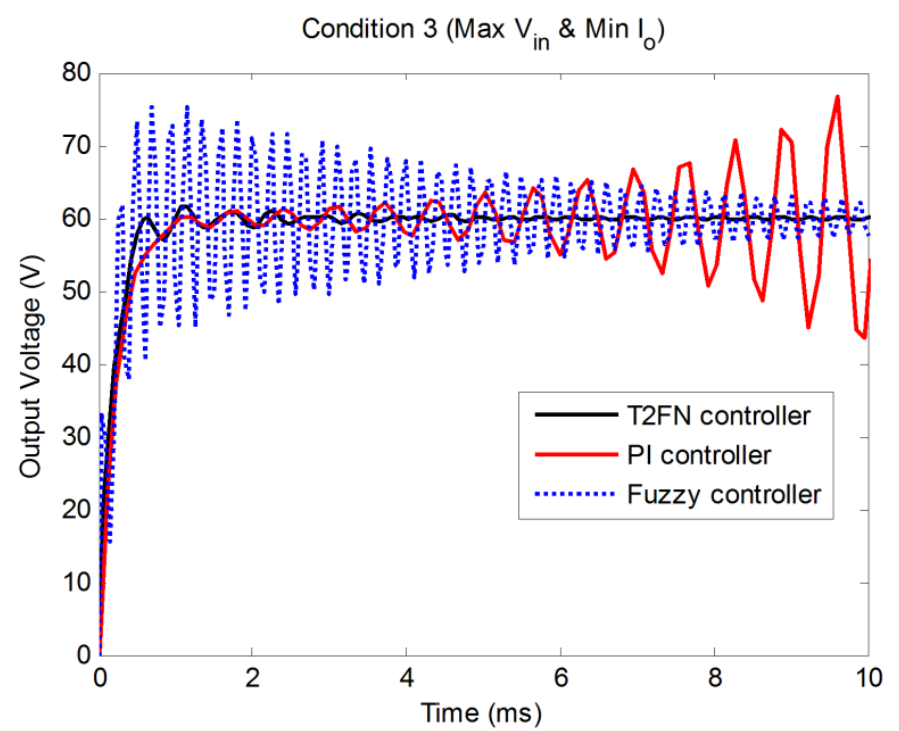

Fig. 10. Response for condition $3\left(V_{i n}=13 v, I_{0}=2.16 \mathrm{~mA}, R=6 K \Omega\right)$

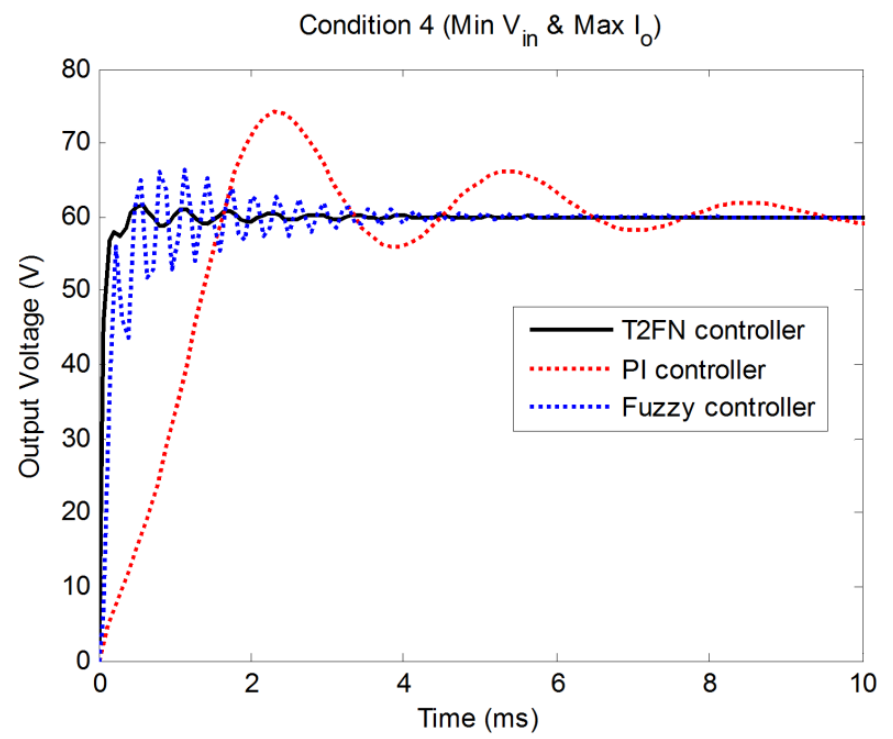

Fig. 11. Response for condition $4\left(V_{\text {in }}=6 v, I_{o}=4 m A, R=1.5 \mathrm{~K} \Omega\right)$

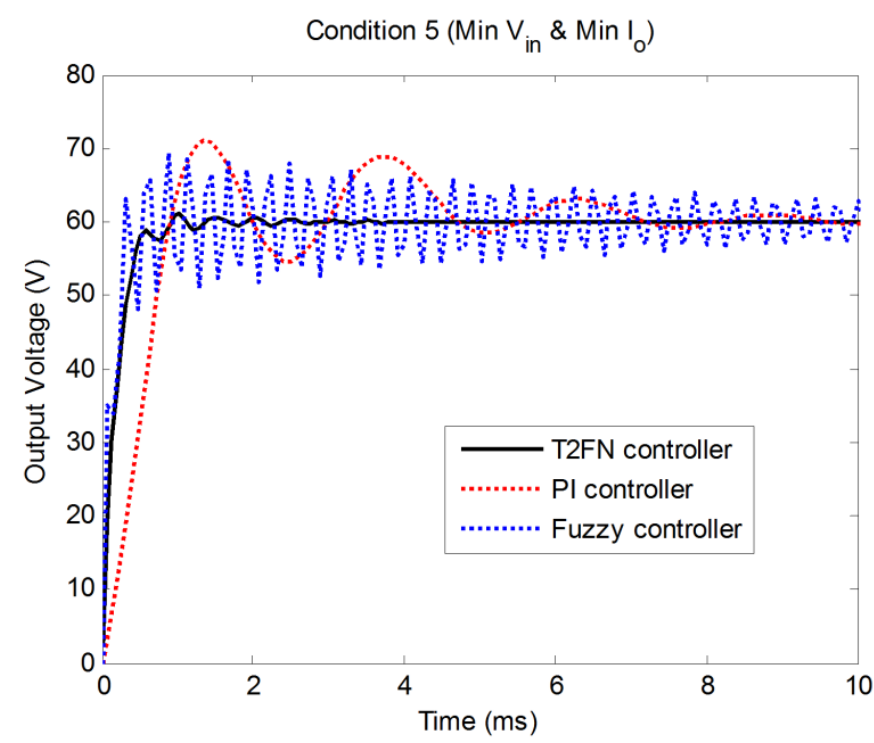

Fig. 12. Response for condition $5\left(V_{\text {in }}=6 v, I_{0}=1 \mathrm{~mA}, R=6 K \Omega\right)$ 
According to Figs. 8-12, the proposed T2FN controller can provide significant improvements in case of dynamic response of ultra-lift DC/DC converter comparing to PI controller and fuzzy controllers under all given operational conditions. In addition, it has more stable dynamic response than other employed controllers.

In Fig. 10, dynamic response of T2FNC is stable and suitable for this ultra-lift DC/DC converter; while the response of fuzzy controller is a damped oscillation. Moreover, it can be seen that the PI controller is totally unstable for this case and the corresponding dynamic response is an undamped oscillation, which is because that the small signal approach is used to design PI controller for nonlinear system. In this small signal approach, the nonlinear system is linearized at an operation point around nominal conditions. Hence, the parameters of PI controller are computed according to nominal conditions. It is obvious that the performance of PI controller is undesirable when real operational condition is completely different from nominal condition (such as condition 3 ).

Moreover, the value of overshoot and settling time for three proposed controllers in five assumed operating conditions are compared in Table 5 and Table 6.

Table 5. The value of \%overshoot for three proposed controllers under five operating conditions.

\begin{tabular}{|c|c|c|c|}
\hline Condition number & T2FNC & Fuzzy controller & PI controller \\
\hline 1 & $2.11 \%$ & $8.03 \%$ & $17.96 \%$ \\
\hline 2 & $3.09 \%$ & $17.90 \%$ & $21.03 \%$ \\
\hline 3 & $4.32 \%$ & $25.21 \%$ & Non \\
\hline 4 & $4.17 \%$ & $11.03 \%$ & $23.12 \%$ \\
\hline 5 & $3.15 \%$ & $15.12 \%$ & $17.06 \%$ \\
\hline
\end{tabular}

Table 6. The value of settling time (ms) for three proposed controllers under five operating conditions.

\begin{tabular}{|c|c|c|c|}
\hline Condition number & T2FNC & Fuzzy controller & PI controller \\
\hline 1 & 4.8 & 5.4 & 8.7 \\
\hline 2 & 6.7 & 8.8 & 10.3 \\
\hline 3 & 6.2 & 15.2 & Non \\
\hline 4 & 5.5 & 6.1 & 11.5 \\
\hline 5 & 3.9 & 14.1 & 10.7 \\
\hline
\end{tabular}

According to Table 5 and Table 6, overshoot and settling time for dynamic response of T2FNC are less than that of the fuzzy and PI controllers under the five assumed operating conditions. Therefore, the T2FN controller has more desirable performance than other proposed controllers and it can improve transient stability and dynamic responses of the ultra-lift DC/DC converter. 


\section{Conclusion}

Due to the uncertainty in structure and electrical elements of ultra-lift DC-DC converter and also nonlinear performance of this converter, designing an effective controller is a challenging task. This paper employs a T2FN controller to improve the transient stability and dynamic response of an ultralift DC-DC converter under different operation conditions. Moreover, a new evolutionary optimization technique called firefly algorithm has been applied for tuning the T2FNC parameters.

The performance of T2FNC has been investigated at different operating points. In addition, it has been compared with fuzzy and PI controllers in order to demonstrate its effectiveness.

The obtained results have shown that the T2FN controller has more desirable performance than other proposed controllers in terms of faster dynamic response, better rejection of disturbances and less settling time and peak overshoot. Thus the proposed methodology can improve transient stability and dynamic respond of the ultra-lift DC/DC converter. Moreover, the T2FNC controller can properly handle uncertainties associated with structure of converter, measured control signals and measuring devices. Hence, it is useful to improve the performance of ultra-lift DC-DC converters in many renewable energy applications.

\section{References}

[1] M. Heidari, T. Balachandran, V. Aravinthan, V. Namboodiri, and G. Chen, "ALARM: average low-latency medium access control communication protocol for smart feeders," IET Generation, Transmission \& Distribution, vol. 10, no. 11, pp. 2647-2657, 2016.

[2] J. Olamaei, M. A. Ghasemabadi, and M. H. Kapourchali, "An efficient method for load flow analysis of distribution networks including PV nodes," in Electric Power and Energy Conversion Systems (EPECS), 2011 2nd International Conference on, 2011, pp. 1-6: IEEE.

[3] H. Aburub, M. Heidari-Kapourchali, W. T. Jewell, and V. Aravinthan, "Performance characterization of a solar panel: A case study," in North American Power Symposium (NAPS), 2014, 2014, pp. 1-4: IEEE.

[4] M. H. Kapourchali and M. Sepehry, "Fault Detector and Switch Placement in Cyber-Enabled Power Distribution Network," IEEE Transactions on Smart Grid, 2016.

[5] S. H. Gilani, H. Afrakhte, and M. J. Ghadi, "Probabilistic method for optimal placement of wind-based distributed generation with considering reliability improvement and power loss reduction," in Thermal Power Plants (CTPP), 2012 4th Conference on, 2012, pp. 1-6: IEEE.

[6] S. H. Gilani, M. J. Ghadi, and H. Afrakhte, "Optimal allocation of wind turbines considering different costs for interruption aiming at power loss reduction and reliability improvement using imperialistic competitive algorithm," International Review of Electrical Engineering, vol. 8, no. 1, pp. 284-296, 2013.

[7] S. Sivakumar, M. J. Sathik, P. Manoj, and G. Sundararajan, "An assessment on performance of DC-DC converters for renewable energy applications," Renewable and Sustainable Energy Reviews, vol. 58, pp. 1475-1485, 2016. 
[8] B. Banerjee, R. M. Kotecha, and W. W. Weaver, "Digital memory look-up based implementation of sliding mode control for dc-dc converters," Control Engineering Practice, vol. 54, pp. 1-11, 2016.

[9] D. J. Pradeep, M. M. Noel, and N. Arun, "Nonlinear control of a boost converter using a robust regression based reinforcement learning algorithm," Engineering Applications of Artificial Intelligence, vol. 52, pp. 1-9, 2016.

[10] U. Sadek, A. Sarjaš, A. Chowdhury, and R. Svečko, "FPGA-based optimal robust minimal-order controller structure of a DC-DC converter with Pareto front solution," Control Engineering Practice, vol. 55, pp. 149-161, 2016.

[11] A. G. Saravanan and M. Rajaram, "Fuzzy controller for dynamic performance improvement of a half-bridge isolated DC-DC converter," Neurocomputing, vol. 140, pp. 283-290, 2014.

[12] P. Siano and C. Citro, "Designing fuzzy logic controllers for DC-DC converters using multi-objective particle swarm optimization," Electric Power Systems Research, vol. 112, pp. 74-83, 2014.

[13] L. Guo, J. Y. Hung, and R. Nelms, "Design of a fuzzy controller using variable structure approach for application to DC-DC converters," Electric Power Systems Research, vol. 83, no. 1, pp. 104-109, 2012.

[14] L. Guo, J. Y. Hung, and R. Nelms, "Comparative evaluation of sliding mode fuzzy controller and PID controller for a boost converter," Electric Power Systems Research, vol. 81, no. 1, pp. 99-106, 2011.

[15] C. Elmas, O. Deperlioglu, and H. H. Sayan, "Adaptive fuzzy logic controller for DC-DC converters," Expert Systems with Applications, vol. 36, no. 2, pp. 1540-1548, 2009.

[16] A. M. El-Nagar and M. El-Bardini, "Interval type-2 fuzzy neural network controller for a multivariable anesthesia system based on a hardware-in-theloop simulation," Artificial intelligence in medicine, vol. 61, no. 1, pp. 1-10, 2014.

[17] O. Castillo and P. Melin, "A review on interval type-2 fuzzy logic applications in intelligent control," Information Sciences, vol. 279, pp. 615-631, 2014.

[18] E. Yesil, "Interval type-2 fuzzy PID load frequency controller using Big Bang-Big Crunch optimization," Applied Soft Computing, vol. 15, pp. 100$112,2014$.

[19] A. Sharifian and S. Sharifian, "A new power system transient stability assessment method based on type-2 fuzzy neural network estimation," International Journal of Electrical Power \& Energy Systems, vol. 64, pp. 7187, 2015.

[20] R. H. Abiyev, O. Kaynak, and E. Kayacan, "A type-2 fuzzy wavelet neural network for system identification and control," Journal of the Franklin Institute, vol. 350, no. 7, pp. 1658-1685, 2013.

[21] E. Kayacan, Y. Oniz, A. C. Aras, O. Kaynak, and R. Abiyev, "A servo system control with time-varying and nonlinear load conditions using type-2 TSK fuzzy neural system," Applied Soft Computing, vol. 11, no. 8, pp. 5735-5744, 2011.

[22] F. L. Luo and H. Ye, Essential DC/DC Converters. CRC Press, 2005.

[23] M. H. Rashid, Power electronics handbook: devices, circuits and applications. Academic press, 2010.

[24] N. N. Karnik and J. M. Mendel, "Centroid of a type-2 fuzzy set," Information Sciences, vol. 132, no. 1, pp. 195-220, 2001. 
[25] J. M. Mendel, Uncertain rule-based fuzzy logic systems: introduction and new directions. Prentice Hall PTR Upper Saddle River, 2001.

[26] M. Bakhshipour, M. J. Ghadi, and F. Namdari, "Swarm robotics search \& rescue: A novel artificial intelligence-inspired optimization approach," Applied Soft Computing, 2017.

[27] M. J. Ghadi and A. Baghramian, "A new heuristic method for solving unit commitment problem in competitive environment," International Journal of Soft Computing and Engineering, vol. 2, no. 6, pp. 2231-2307, 2013.

[28] A. Rajabi, L. Li, J. Zhang, J. Zhu, S. Ghavidel, and M. J. Ghadi, "A review on clustering of residential electricity customers and its applications," in Electrical Machines and Systems (ICEMS), 2017 20th International Conference on, 2017, pp. 1-6: IEEE.

[29] A. Azizivahed, S. Ghavidel, M. J. Ghadi, L. Li, and J. Zhang, "Multi-area economic emission dispatch considering load uncertainty," in Electrical Machines and Systems (ICEMS), 2017 20th International Conference on, 2017, pp. 1-6: IEEE.

[30] X.-S. Yang, "Firefly algorithms for multimodal optimization," in International symposium on stochastic algorithms, 2009, pp. 169-178: Springer.

[31] F. L. Luo and H. Ye, "Ultra-lift Luo-converter," IEE Proceedings-Electric Power Applications, vol. 152, no. 1, pp. 27-32, 2005.

[32] F. L. Luo and H. Ye, "Ultra-lift Luo-converter," in Power System Technology, 2004. PowerCon 2004. 2004 International Conference on, 2004, vol. 1, pp. 81-86: IEEE. 